\title{
THE HARDY CLASS OF FUNCTIONS OF BOUNDED BOUNDARY ROTATION
}

\author{
BERNARD PINCHUK ${ }^{1}$
}

\begin{abstract}
The Hardy class for functions of bounded boundary rotation and their derivatives is determined. In the univalent case, a more precise description of the Hardy class in terms of the behavior of the representing measure is obtained.
\end{abstract}

1. Introduction. $V_{k}$ denotes the class of normalized analytic functions in $|z|<1$ having boundary rotation bounded by $k \pi$ and $S_{k}$ denotes the subclass of $V_{k}$ consisting of univalent functions.

In $\S \S 2$ and 3 we provide precise definitions of the classes and describe how certain aspects of the behavior of the function depend on the representing measure. Following this, we use known results for convex functions to determine the Hardy class of $V_{k}$ functions and their derivatives. In $\$ 5$ we determine the Hardy class for $S_{k}$ functions and display how it depends on the behavior of the representing measure. We conclude with coefficient estimates.

2. The class $V_{k}$. We give the most direct analytic definition of the class $V_{k}$.

DefinItION 1. A function $f(z)=z+a_{2} z^{2}+\cdots$, analytic in $|z|<1$, is said to have boundary rotation bounded by $k \pi, k \geqq 2$, if

for $|z|<1$.

$$
\int_{0}^{2 \pi}\left|\operatorname{Re}\left(1+\frac{z f^{\prime \prime}(z)}{f^{\prime}(z)}\right)\right| d \theta \leqq k \pi
$$

Here, as throughout, $z=r e^{i \theta}$.

$V_{k}$ denotes the class of all normalized analytic functions in $|z|<1$ having boundary rotation bounded by $k \pi$.

For brevity, we write $1+z f^{\prime \prime}(z) / f^{\prime}(z)=u(z)+i v(z)$.

$V_{k}$ can thus be characterized as the class of normalized analytic functions $f(z)$ in $|z|<1$ for which $u(z)$ is a harmonic function of class $h^{1}$ with $h^{1}$-norm bounded by $k / 2$. It follows (see [2, p. 3]) that $u(z)$ can be written

Received by the editors November 22, 1971 and, in revised form, July 31, 1972.

AMS (MOS) subject classifications (1970). Primary 30A32, 30A78.

Key words and phrases. Boundary rotation, $H^{p}$ space.

${ }^{1}$ Supported in part by National Science Foundation grant GP7952X3.

(C) American Mathematical Society 1973 
as a Poisson-Stieltjes integral:

$$
u(z)=\frac{1}{2 \pi} \int_{0}^{2 \pi} P(r, \theta-t) d \mu(t)
$$

where $\mu(t)$ is of bounded variation and satisfies

$$
\int_{0}^{2 \pi} d \mu(t)=2 \pi, \quad \int_{0}^{2 \pi}|d \mu(t)| \leqq k \pi .
$$

$\mu(t)$ is extended outside the interval $(0,2 \pi]$ so that $\mu(t)-t$ has period $2 \pi$, and is normalized by the conditions $\mu(t)=\frac{1}{2}(\mu(t+0)+\mu(t-0))$ and $\int_{0}^{2 \pi}(\mu(t)-t) d t=0$. With these normalizations, $\mu(t)$ is unique.

Completing (1) to an analytic function, we have the familiar representation for functions in $V_{k}$ :

$$
1+\frac{z f^{\prime \prime}(z)}{f^{\prime}(z)}=\frac{1}{2 \pi} \int_{0}^{2 \pi} \frac{1+z e^{-i t}}{1-z e^{-i t}} d \mu(t)
$$

or

$$
f^{\prime}(z)=\exp \frac{1}{\pi} \int_{0}^{2 \pi}-\log \left(1-z e^{-i t}\right) d \mu(t) .
$$

$\mu(t)$ is called the representing measure for $f(z)$. We now characterize $\mu(t)$ in terms of $f(z)$.

THEOREM 1. Let $f(z) \in V_{k}$ with representing measure $\mu(t)$. Then $\operatorname{Lim}_{r \rightarrow 1} \arg \left\{z f^{\prime}(z)\right\}=\eta(\theta)$ exists and $\eta(\theta)=\mu(\theta)$.

Proof. We have from (3) that

$$
\log f^{\prime}(z)=\frac{1}{\pi} \int_{0}^{2 \pi}-\log \left(1-z e^{-i t}\right) d \mu(t)
$$

Also, $\int_{0}^{2 \pi}-\log \left(1-z e^{-i t}\right) d t=0$. Hence,

$$
\log f^{\prime}(z)=\frac{1}{\pi} \int_{0}^{2 \pi}-\log \left(1-z e^{-i t}\right) d(\mu(t)-t) .
$$

Integrating by parts, we have

$$
\log f^{\prime}(z)=\frac{1}{2 \pi} \int_{0}^{2 \pi} \frac{1+z e^{-i t}}{1-z e^{-i t}}(\mu(t)-t) d t
$$

or

$$
\arg f^{\prime}(z)=\frac{1}{2 \pi} \int_{0}^{2 \pi} P(r, t-\theta)(\mu(t)-t) d t
$$


This is the Poisson integral of a bounded variation function which takes the average value at each jump discontinuity. Hence,

$$
\operatorname{Lim}_{r \rightarrow 1} \arg f^{\prime}\left(r e^{i \theta}\right)=\mu(\theta)-\theta .
$$

This is the assertion of the theorem.

In the case $k=2$, this theorem is well known, see [6, p. 210] and [3, p. 337].

We can write $\mu(t)$ as the difference of two nondecreasing functions, $\mu(t)=p(t)-n(t)$. From (2) it is clear that

$$
\frac{1}{\pi} \int_{0}^{2 \pi} d p(t) \leqq \frac{k}{2}+1 \text { and } \frac{1}{\pi} \int_{0}^{2 \pi} d n(t) \leqq \frac{k}{2}-1 .
$$

It follows, therefore, that we can set

$$
f^{\prime}(z)=\left(g^{\prime}(z)\right)^{[(k / 2)+1] / 2}\left(h^{\prime}(z)\right)^{-[(k / 2)-1] / 2},
$$

where $g(z)$ is a convex function having the representation

$$
g^{\prime}(z)=\exp \frac{1}{\pi} \int_{0}^{2 \pi}-2 \log \left(1-z e^{-i t}\right) d \alpha(t)
$$

where $(k / 2+1) \alpha(t)=p(t)$. A similar representation holds for the convex function $h(z)$. The representation (4) is due to D. A. Brannan [1].

The reader is referred to [4], where the study of these classes was initiated, for the geometric significance.

3. The representing measure. We proved in [5] that for $f(z) \in V_{k}$ with representing measure $\mu(t)$, if $\mu(t)$ concentrates no mass of weight $\geqq \pi$ at $t=\theta$, then $\left|f\left(r e^{i \theta}\right)\right|$ is bounded as $r \rightarrow 1$. We now refine that proof to obtain estimates on $\operatorname{Max}_{|z|=r}|f(z)|$ for $V_{k}$ functions, which depend on the behavior of the representing measure.

THEOREM 2. Let $f(z) \in V_{k}$ and let $\mu(t)$ be the representing measure. If $\pi \alpha, \alpha>1$, is the height of the greatest jump discontinuity of $\mu(t)$, then

for all $\varepsilon>0$.

$$
\underset{|z|=r}{\operatorname{Max}}|f(z)| \leqq C(1-r)^{1-\alpha-\varepsilon}
$$

Proof. Given $\varepsilon>0$ we can find a $\delta>0$ such that $\int_{I} d \mu(t) \leqq \pi(\alpha+\varepsilon)$ for any interval $I$ of length smaller than $2 \delta$. Let $\mu(t)$ have jump of height $\pi \alpha$ 
at $t=t_{0}$. Then,

$$
\begin{aligned}
\left|f^{\prime}\left(r e^{i t_{0}}\right)\right| & =\exp \frac{1}{\pi} \int_{0}^{2 \pi}-\log \left|1-r e^{i\left(t_{0}-t\right)}\right| d \mu(t) \\
& \leqq \exp \frac{1}{\pi}\left\{\int_{t_{0}-\delta}^{t_{0}+\delta}+\int_{t_{0}+\delta}^{t_{0}-\delta}-\log \left|1-r e^{i\left(t_{0}-t\right)}\right| d \mu(t)\right\} \\
& \leqq(1-r)^{-(\alpha+\varepsilon)} \cdot C .
\end{aligned}
$$

$C$ is a constant (depending on $\varepsilon$ ) which bounds the integral over the portion excluding an interval about $t_{0}$.

This bound certainly holds at any other point of $[0,2 \pi]$ as well. Integrating, we have

$$
\left|f\left(r e^{i \theta}\right)\right| \leqq C(1-r)^{1-(\alpha+\varepsilon)} .
$$

4. The means $M_{\eta}(r, f)$. Let

$$
M_{p}(r, f)=\left(\frac{1}{2 \pi} \int_{0}^{2 \pi}\left|f\left(r e^{i \theta}\right)\right|^{p} d \theta\right)^{1 / p}, \quad 0<p<\infty
$$

and let

$$
M(r, f)=\underset{|z|=r}{\operatorname{Max}}|f(z)| .
$$

Analytic functions in $|z|<1$ for which $M_{p}(r, f)$ is bounded as $r \rightarrow 1$ are said to be of class $H^{p}$.

It is well known [2, p. 88] that if $f^{\prime}(z) \in H^{p}, 0<p \leqq 1$, then $f(z) \in H^{p /(1-p)}$.

For univalent functions, we have (see [2, p. 51]):

$$
M_{p}^{p}(r, f) \leqq \frac{1}{p} \int_{0}^{r} \frac{(M(R, f))^{p}}{R} d R .
$$

TheOREM 3. Let $f(z) \in V_{k}$. Then, $f^{\prime}(z) \in H^{p}$ for all $p<2 /(k+2)$ and $f(z) \in H^{p}$ for all $p<2 / k$. Furthermore, if $f^{\prime}(z)$ is not of the form

$$
\left(1-z e^{-i t_{0}}\right)^{-[(k / 2)+1]} \cdot \exp \frac{1}{\pi} \int_{0}^{2 \pi}-\log \left(1-z e^{-i t}\right) d n(t),
$$

then $f^{\prime}(z) \in H^{p}$ for some $p>2 /(k+2)$ and $f(z) \in H^{p}$ for some $p>2 / k$.

We note that this theorem is best possible. Indeed, the function

$$
f(z)=\frac{1}{k}\left[\left(\frac{1+z}{1-z / 2}-1\right]\right.
$$

belongs to $V_{k}$, yet $f(z) \notin H^{(2 / k)}$ and $f^{\prime}(z) \notin H^{2 /(k+2)}$. 
Proof. By (4) we have

$$
\begin{aligned}
\int_{0}^{2 \pi}\left|f^{\prime}\left(r e^{i \theta}\right)\right|^{p} d \theta & =\int_{0}^{2 \pi}\left|g^{\prime}\left(r e^{i \theta}\right)\right|^{p[(k / 2)+1] / 2}\left|h^{\prime}\left(r e^{i \theta}\right)\right|^{-p[(k / 2)-1] / 2} d \theta \\
& \leqq C \int_{0}^{2 \pi}\left|g^{\prime}\left(r e^{i \theta}\right)\right|^{p[(k / 2)+1] / 2} d \theta .
\end{aligned}
$$

The inequality follows from the distortion theorem $\left|h^{\prime}\left(r e^{i \theta}\right)\right| \geqq(1+|z|)^{-2}$ for convex functions. Since $g^{\prime}(z) \in H^{p}$ for $p<\frac{1}{2}$, we conclude that $f^{\prime}(z) \in H^{p}$ for $p<2 /(k+2)$. It now follows that $f(z) \in H^{p}$ for $p<2 / k$.

To prove the second assertion, we note that if $f^{\prime}(z)$ is not of the form (7), then in the decomposition (4), $g(z)$ is not of the form

$$
g(z)=z\left(1-z e^{-i t_{0}}\right)^{-1} .
$$

If $g(z)$ is not of this form, then by [3, Theorem 3], there exists a $\delta>0$ such that $g^{\prime}(z) \in H^{1 / 2+\delta}$. Using this information in (8), we have the desired conclusion.

5. The univalent case. We now use Theorem 2 together with the inequality (6) to determine the Hardy class for univalent $V_{k}$ functions. We denote the set of all univalent functions in $V_{k}$ by $S_{k}$. Recall, that for $2 \leqq k \leqq 4, V_{k}=S_{k}$.

THEOREM 4. Let $f(z) \in S_{k}$ with representing measure $\mu(t)$. If $\pi \alpha, \alpha>1$, is the height of the greatest positive jump discontinuity of $\mu(t)$, then $f(z) \in H^{p}$ for all $p<(\alpha+\varepsilon-1)^{-1}$ for all $\varepsilon>0$.

The height of the greatest positive jump of $\mu(t)$ is $\pi(k / 2+1)$, and this occurs only for a function of the form (7). For the case $2 \leqq k \leqq 4$, this provides a second proof of the portion of Theorem 3 referring to the Hardy class of $f(z)$.

A theorem of Hardy and Littlewood [2, p. 98] asserts that if $f(z) \in H^{p}$, $0<p \leqq 1$, the Taylor coefficients of $f(z)$ satisfy $a_{n}=o\left(n^{1 / p-1}\right)$. We thus have the following coefficient estimates.

THEOREM 5. Let $f(z) \in V_{k}$, with Taylor coefficients $a_{n}$. Then

(1) $a_{n}=o\left(n^{1 / p-1}\right)$ for $p<2 / k$.

(2) Iff $f^{\prime}(z)$ is not of the form (7), then $a_{n}=o\left(n^{k / 2-1}\right)$. Indeed, $a_{n}=o\left(n^{k / 2-1-\varepsilon}\right)$ for some $\varepsilon>0$.

(3) Iff $(z)$ is univalent and if $\pi \alpha, \alpha>1$, is the height of the greatest positive jump of the representing measure, then $a_{n}=o\left(n^{\alpha+\varepsilon-2}\right)$. 
The estimate (1) also follows from Robertson's estimate [7], $a_{n}=$ $O\left(n^{k / 2-1}\right)$. (2) provides a refinement of this, and (3) even more so if $2 \leqq k \leqq 4$.

We thank the referee for bringing [1] to our attention.

\section{REFERENCES}

1. D. A. Brannan, On functions of bounded boundary rotation. 1, Proc. Edinburgh Math. Soc. (2) 16 (1968/69), 339-347. MR 41 \#8642.

2. P. L. Duren, Theory of $H^{p}$ spaces, Pure and Appl. Math., vol. 38, Academic Press, New York, 1970. MR 42 \#3552.

3. P. J. Eenigenburg and F. R. Keogh, The Hardy class of some univalent functions and their derivatives, Michigan Math. J. 17 (1970), 335-346.

4. V. Paatero, Über die konforme Abbildung von Gebieten deren Ränder von beschränkter Drehung sind, Ann. Acad. Sci. Fenn. A 33 (1931), no. 9.

5. B. Pinchuk, Functions of bounded boundary rotation, Israel J. Math. (10) 1 (1971), 6-16.

6. Ch. Pommerenke, On starlike and convex functions, J. London Math. Soc. 37 (1962), 209-224. MR 25 \#1279.

7. M. S. Robertson, Coefficients of functions with bounded boundary rotation, Canad. J. Math. 21 (1969), 1477-1482. MR 41 \#458.

School of Mathematics, Institute for Advanced Study, Princeton, New JERSEY 08540

Current address: Department of Mathematics, Bar-Ilan University, Ramat-Gan, Israel 